\title{
On the determinants of surface brightness
}

\author{
SERGIO CESARE MASIN \\ University of Padua, Padua, Italy
}

\begin{abstract}
The brightness of an achromatic surface with luminance $S$ on an achromatic background with luminance $B$ varies with $S$, with $B$, and with the luminance step $\Delta L$ at the border of the surface. In agreement with previous findings indicating that the visual system can perform as a photometer, the results of the two experiments reported here show that $S$ and $B$ determined surface brightness independently of $\Delta L$ when the surface was adjacent to and when it was separated from the background. This finding suggests that surface brightness depends on the integration of neural signals representing magnitudes of absolute luminance. A weighted-averagemodel of this integration is proposed.
\end{abstract}

In the present study, the luminance determinants of surface brightness were explored. The findings that uniform surfaces vanish when their retinal contour is stabilized and that on-center and off-center visual neurons signal the luminance step at the border of surfaces support the current view that this luminance step determines surface brightness (Arend, Buehler, \& Lockhead, 1971; Fiorentini, Baumgartner, Magnussen, Schiller, \& Thomas, 1990; Grossberg \& Todorović, 1988; Krauskopf, 1963; Land \& McCann, 1971; Ross \& Pessoa, 2000). However, here it is shown that a reanalysis of the experimental results reported by Beck (1974) and Kozaki (1973) reveals that absolute luminances determine surface brightness independently of the luminance step at the border of surfaces.

\section{EXPERIMENT 1}

Experiment 1 was designed to replicate the results of Beck (1974) and Kozaki (1973) using a broader range of stimulus parameters.

Surfaces presented in a dark field were used. Since, in these surfaces, whiteness matches brightness (Redding \& Lester, 1980) and is complementary to blackness (Quinn, Wooten, \& Ludman, 1985), the effects of absolute luminances on each of these attributes were tested. ${ }^{1}$

\section{Method}

Participants. Forty-eight students of the University of Padua with normal or corrected-to-normal vision participated to fulfill a course requirement.

Stimuli. In a dark room, each stimulus appeared on the frontal parallel $33 \times 25 \mathrm{~cm}$ screen of an Apple monitor controlled by a Macintosh 7200 computer. The viewing distance was about $80 \mathrm{~cm}$.

The stimulus was a $4.5 \times 4.5 \mathrm{~cm}$ achromatic square in the middle of an achromatic background covering the entire screen. Let $S$ and $B$ denote the luminance of the square and that of the background, respectively. There were 30 stimuli, one for each different

Correspondence should be addressed to S. C. Masin, Department of General Psychology, University of Padua, 35131 Padova, Italy (e-mail: scm@unipd.it). pair of $S$ and $B$, in which $S$ was $10,20,40,60$, or $80 \mathrm{~cd} / \mathrm{m}^{2}$ and $B$ was $1,15,30,50,70$, or $110 \mathrm{~cd} / \mathrm{m}^{2}$. The stimuli remained on the screen until the experimenter typed the participant's response. The intertrial interval was $3 \mathrm{sec}$.

Procedure. The experiment was divided into three sessions. During presentation of the instructions for each session, a horizontal row of eight equidistant $2.2 \times 2.2 \mathrm{~cm}$ achromatic squares with luminances of $0.05,5,10,20,40,70,100$, and $140 \mathrm{~cd} / \mathrm{m}^{2}$, increasing from left to right, was displayed on a background with $B=15 \mathrm{~cd} / \mathrm{m}^{2}$. In the first session, the participants rated the perceived amount of light coming from the square of each stimulus using integers from 0 to 100 , with 0 representing the brightness of the leftmost square and 100 that of the rightmost square in the row of eight squares. In the remaining two sessions, the participants rated the perceived amount either of whiteness or of blackness in the square of each stimulus using integers from 0 to 100 , with 0 representing the absence of white or of black and 100 representing the presence only of white or only of black. Half of the participants rated whiteness in the second session and blackness in the third. For the other half, the order was reversed. In each session, the series of 30 stimuli was shown two times consecutively, each time with the stimuli in random order.

\section{Results}

For each stimulus, the mean of the two ratings produced by a participant during each session was used as an individual score. Figures $1 \mathrm{~A}$ to $1 \mathrm{C}$ show the mean ratings of brightness, whiteness, and blackness, respectively, as a function of $S$ for each $B$.

A 2 (brightness vs. whiteness) $\times 5(S) \times 6(B)$ analysis of variance (ANOVA) showed that the effect of brightness versus whiteness and the related interactions were not significant $[F(1,47)=0.01, F(4,188)=1.8, F(5,235)=$ 1.9 , and $F(20,940)=1.2$, respectively], confirming that brightness matches whiteness in surfaces presented in a dark field (Redding \& Lester, 1980). The effects of $S$ $[F(4,188)=1,282]$ and of $B[F(5,235)=614]$ and the interaction of these factors $[F(20,940)=12]$ were significant at the .001 level, confirming that the brightness of the surface varied with $S$ and $B$ (Beck, 1974; Kozaki, 1973).

With each score for blackness subtracted from 100, a 2 (whiteness vs. blackness) $\times 5(S) \times 6(B)$ ANOVA 
showed that the effects of all factors $[F(1,47)=64$, $F(4,188)=1,207$, and $F(5,235)=632$, respectively], the interactions between whiteness-versus-blackness and $S$ $[F(4,188)=7.3]$ and between $S$ and $B[F(20,940=25]$, and the three-factor interaction $[F(20,940=3.7]$ were significant at the .001 level, whereas the interaction between whiteness-versus-blackness and $B[F(5,235=0.6]$ was not significant. Thus, whiteness and blackness were moderately noncomplementary. ${ }^{2}$

Let the luminance step at the border of the square be $\Delta L=|S-B|$. In Figure $1 \mathrm{~A}$, for each $B$ from 15 to $70 \mathrm{~cd} / \mathrm{m}^{2}$, each cross shows the point interpolated linearly between the data points corresponding to the two $S$ s closest to $B$. The slope of the line fitting these interpolated points was significantly different from zero $[F(3,141)=105, p<.0005]$. Each interpolated point represents an estimate of the value to which mean rated brightness would have tended if $\Delta L$ had tended to 0 $(S=B)$. Since this value corresponds to $\Delta \mathrm{L}=0$ and increases with $\mathrm{S}=\mathrm{B}, \mathrm{S}$ and $\mathrm{B}$ must have determined surface brightness independently of $\Delta \mathrm{L}$. The results for whiteness and blackness confirm this conclusion. For each $B$ from 15 to $70 \mathrm{~cd} / \mathrm{m}^{2}$, each cross in the respective Figures $1 \mathrm{~B}$ and $1 \mathrm{C}$ shows the linearly interpolated point representing an estimate of the value to which mean rated whiteness and mean rated blackness would have tended if $\Delta L$ had tended to 0 . The slope of the line fitting the interpolated points for whiteness and that of the line fitting the interpolated points for blackness were significantly different from zero $\left[F_{\mathrm{s}}(3,141)=117\right.$ and 125 , respectively; $p<.0005]$. The interpolated value of mean rated whiteness increases as $S=B$ increases, and the interpolated value of mean rated blackness decreases as $S=B$ increases, in agreement with the fact that whiteness matches brightness and is almost complementary to blackness.

\section{Discussion}

The demonstration that $S$ and $B$ determined surface brightness independently of $\Delta L$ implies that the visual system contained the neural representations of the magnitudes of these absolute luminances. I propose that the integration of these representations occurs according to the weighted-average model:

$$
\beta=w_{0} S+w_{1}\left(k_{1}-B\right)
$$

where $\beta$ is the amount of brightness of the square due to the integrated effect of absolute luminances, and $w_{0}, w_{1}$, and $k_{1}$ are unknown positive parameters. This model does not exclude the possibility that $\Delta L$ may also affect $\beta$.

In Figure 1A, the trend of crosses is roughly linear with a positive slope. The fact that this slope is positive means that $w_{0}>w_{1}$. Given that brightness increases as $S$ increases and decreases as $B$ increases (Figure 1A), the relation $w_{0}>$ $w_{1}$ means that the brightness-increment rate due to $S$ is higher than the brightness-decrement rate due to $B$.

\section{EXPERIMENT 2}

On the background mentioned above, consider a disk whose border is the inner border of a ring. Let $\Delta L=\mid S-$ $R$, where $S$ is the luminance of the disk and $R$ is that of the ring. With $R$, the present weighted-average model contends that the amount of brightness of the disk due to the integrated effect of absolute luminances is

$$
\beta=w_{0} S+w_{1}\left(k_{1}-B\right)+w_{2}\left(k_{2}-R\right),
$$

where $\beta$ is the brightness of the disk, $w_{0}, w_{1}$, and $k_{1}$ are as described above, and $w_{2}$ and $k_{2}$ are additional unknown positive parameters. Reid and Shapley (1988) found that $B$ affects the brightness of the disk in spite of the ring. This finding is in agreement with Equation 2, because it shows that $B$ affects surface brightness inde-
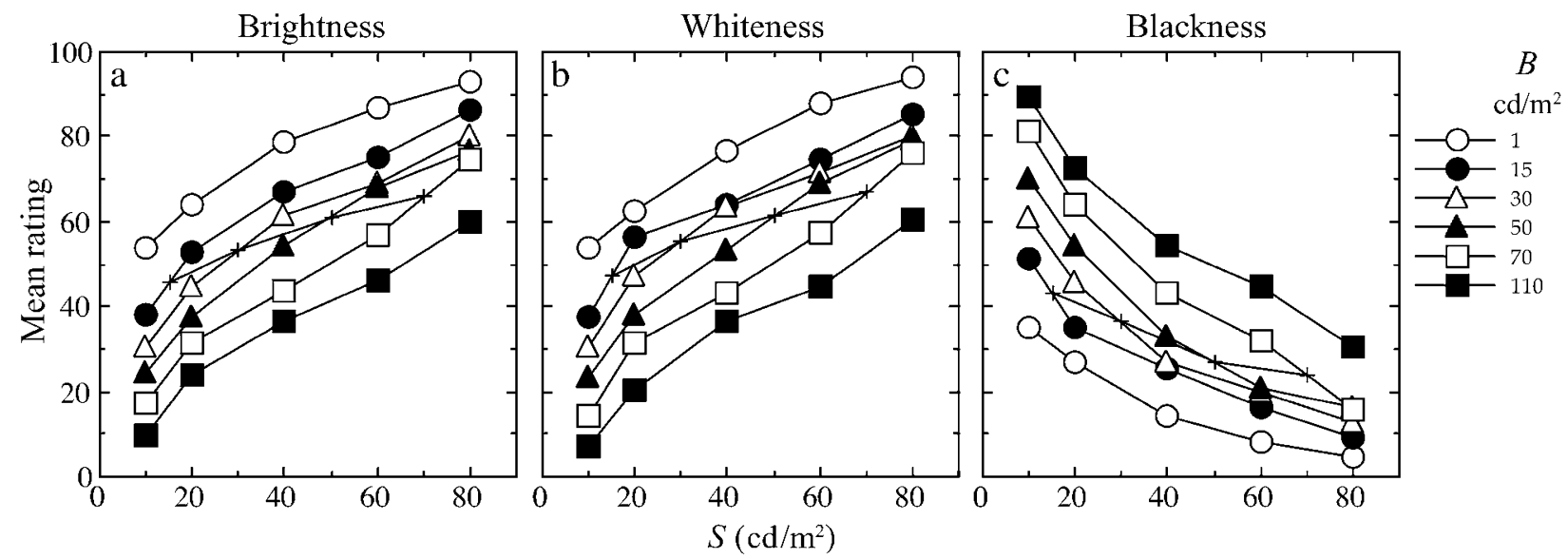

Figure 1. Results of Experiment 1 for an achromatic square with luminance $S$ on an achromatic background with luminance $B$ : mean ratings of (a) brightness, (b) whiteness, and (c) blackness as a function of $S$, for each $B$. Crosses indicate estimates of mean ratings tend $S=B$. 
pendently of $\Delta L$ also when a surface is surrounded by another surface. For $S=B$, Equation 2 implies that

$$
\beta=\left[k_{1} w_{1}+w_{2}\left(k_{2}-R\right)\right]+\left(w_{0}-w_{1}\right) S,
$$

where the quantity in brackets is constant for each $R$. For each $R$, Equation 3 predicts that $\beta$ increases with $S=B$ because $w_{0}>w_{1}$. The present experiment served to verify this prediction.

\section{Method}

The method was the same as that of the first session of Experiment 1 , with the following exceptions.

Participants. Eleven students of the University of Padua with normal or corrected-to-normal vision participated for pay. These participants had not taken part in Experiment 1.

Stimuli. In a dark room, each stimulus appeared in the middle of an achromatic background covering the entire monitor screen. The stimulus was an achromatic disk whose border was the inner border of an achromatic ring. The inner and outer diameters of the ring were 3 and $6 \mathrm{~cm}$, respectively. With $S, R$, and $B$ denoting the luminances of disk, ring, and background, respectively, the values for $S$ and for $B$ were those used in Experiment 1 for the square and for the background, respectively, and those for $R$ were 5,55 , and $105 \mathrm{~cd} / \mathrm{m}^{2}$. There were 90 stimuli, one for each different combination of these luminances.

Procedure. The participants rated the brightness of the disk. The series of the 90 stimuli was shown three times consecutively, each time with the stimuli in random order.

\section{Results and Discussion}

For each stimulus, the mean of the three ratings produced by a participant was used as an individual score. For each $R$ and for each $B$, Figures $2 \mathrm{~A}$ to $2 \mathrm{C}$ show mean rated brightness as a function of $S$.

A $3(R) \times 5(S) \times 6(B)$ ANOVA showed that the effects of all factors $[F(2,20)=62, F(4,40)=389$, and $F(5,50)=305$, respectively $]$ and all interactions $[S \times R$ : $F(8,80)=8 ; S \times B: F(20,200)=7 ; R \times B: F(10,100)=$ 10 ; and $S \times R \times B: F(40,400)=2.5$ ] were significant at the .001 level. These results confirm that $S, B$, and $R$ si- multaneously determined the brightness of the disk. Since the ring separated the disk from the background, these results confirm that $B$ affected surface brightness independently of $\Delta L$ (Reid \& Shapley, 1988; Sugita, 1995; Sugita \& Mimura, 1991).

We have seen that Equation 3 predicts that, for each $R$, the brightness of the disk increases with $S=B$ because $w_{0}>w_{1}$. In Figure 2, for each $R$ and for each $B$ from 15 to $70 \mathrm{~cd} / \mathrm{m}^{2}$, each cross shows the linearly interpolated point representing an estimate of mean rated brightness for $S=B$. For each $R$, the slope of the line fitting these interpolated points is statistically different from zero $\left[F_{\mathrm{S}}(3,30)=99,68\right.$, and 64 , respectively; $\left.p<.0005\right]$. As was predicted, the interpolated value of mean rated brightness increased with $S=B$ for each $R$.

\section{GENERAL DISCUSSION}

The present results indicate that absolute luminances determined surface brightness independently of $\Delta L$, without excluding the possibility that $\Delta L$ could also influence this perception. Studies on the brightness of Ganzfelds indicate that the visual system can perform as a photometer (Barlow, 1991; Barlow, Snodderly, \& Swadlow, 1978; Barlow \& Verillo, 1976; Bolanowski, 1987; Corwin \& Green, 1978; Knau, 2000; Papaioannou, 1974). In line with this evidence, the present results imply that magnitudes of absolute luminance are represented by neural signals. Multiple neural signals representing magnitudes of absolute luminance must be integrated to produce a single surface brightness. This integration could depend on the complexity of the stimulus and on the overall level of illumination of the field. Equations 1 and 2 encode a possible rule for this integration for simple stimuli in a dark field.

In humans and monkeys, neural representations of magnitude of absolute luminances are available in the lateral geniculate nucleus (Barlow, 1991). Using modu-
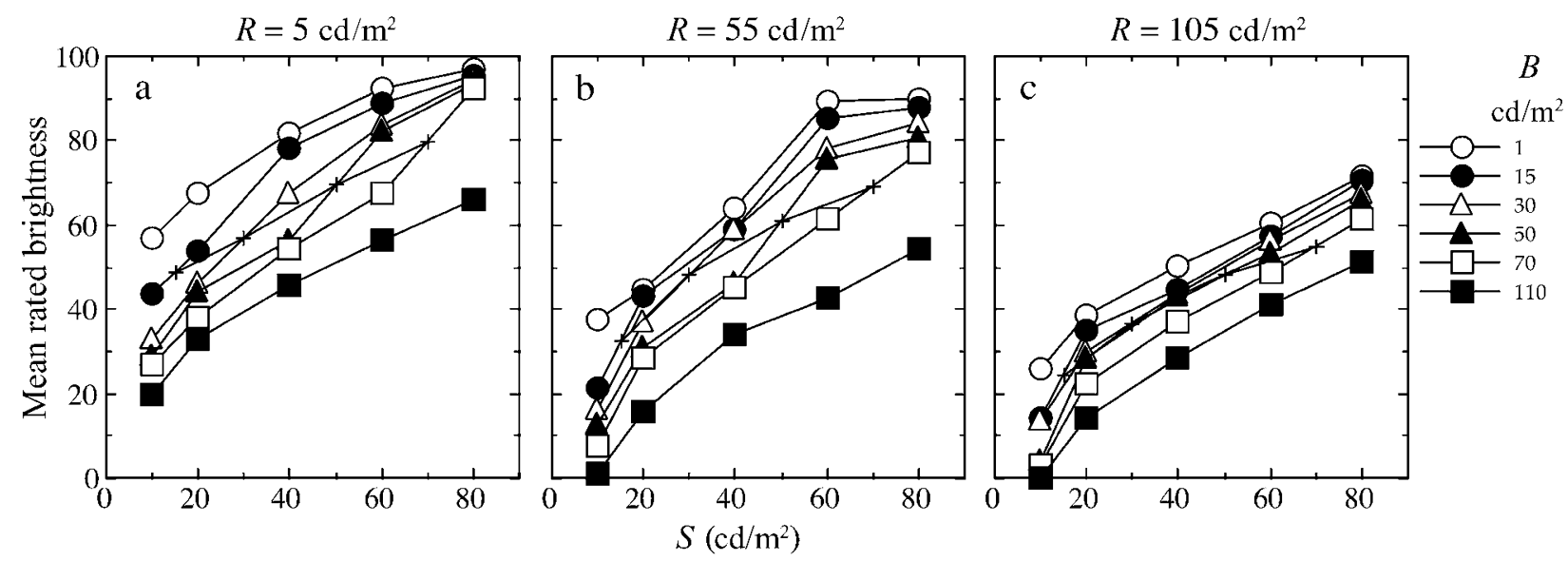

Figure 2. Results of Experiment 2 for an achromatic disk with luminance $S$ enclosed within an achromatic ring with luminance $R$ on an achromatic background with luminance $B$ : mean ratings of brightness of the disk as a function of $S$, for each $R$ and for each $B$. Crosses indicate estimates of mean rated brightness for $S=B$. 
lated context luminances that either changed or did not change the brightness of lights projected on cat retina, Rossi and Paradiso (1999) and Rossi, Rittenhouse, and Paradiso (1996) found that, in the optic tract, responses of retinal ganglion cells did not correlate with changes in brightness, whereas responses of many neurons in the striate cortex and of a small fraction of neurons in the lateral geniculate nucleus correlated with changes in brightness. Consequently, it is possible that signals representing magnitudes of absolute luminance from the lateral geniculate nucleus are integrated in the striate cortex to produce representations of surface brightness. Shevell, Holliday, and Whittle (1992) and Sugita and Mimura (1991) found that $B$ does not affect the brightness of a disk such as that in Experiment 2 when the disk is viewed haploscopically. It follows that, when the disk is viewed directly, $B$ affects the brightness of the disk at the level of the binocular neural representation of the retinal images. This finding confirms that the integration of neural signals representing magnitudes of absolute luminance occurs at a late stage of the neural processing of luminance information.

\section{REFERENCES}

Arend, L. E., Buehler, J. N., \& Lockhead, G. R. (1971). Difference information in brightness perception. Perception \& Psychophysics, $\mathbf{9}$, 367-370.

BARLOW, R. B. (1991). Brightness sensation and the neural coding of light intensity. In S. J. Bolanowski \& G. A. Gescheider (Eds.), Ratio scaling of psychologicalmagnitude: In honor of S. S. Stevens (pp. 163182). Hillsdale, NJ: Erlbaum.

Barlow, R. B., Snodderly, D. M., \& Swadlow, H. A. (1978). Intensity coding in primate visual system. Experimental Brain Research, 31, 163-177.

Barlow, R. B., \& Verillo, R. T. (1976). Brightness sensation in a Ganzfeld. Vision Research, 16, 1291-1297.

BECK, J. (1974). Dimensions of an achromatic surface color. In R. B. MacLeod \& H. L. Pick (Eds.), Perception: Essays in honor of James J. Gibson (pp. 166-184). Ithaca, NY: Cornell University Press.

Bolanowski, S. J. (1987). Contourless stimuli produce binocular brightness summation. Vision Research, 27, 1943-1951.

Corwin, T. R., \& Green, M. A. (1978). The Broca-Sulzer effect in a Ganzfeld. Vision Research, 18, 1675-1678.

Curtis, D. W., \& Rule, S. J. (1978). Judgments of average lightness and darkness: A further consideration of inverse attributes. Perception \& Psychophysics, 24, 343-348.

Fiorentini, A., Baumgartner, G., Magnussen, S., Schiller, P. H., \& Thomas, J. P. (1990). The perception of brightness and darkness: Relations to neuronal receptive fields. In L. Spillmann \& J. S. Werner (Eds.), Visual perception: The neurophysiological foundations (pp. 129-161). San Diego: Academic Press.

Grossberg, S., \& Todorović, D. (1988). Neural dynamics of 1-D and 2-D brightness perception: A unified model of classical and recent phenomena. Perception \& Psychophysics, 43, 241-277.

HegGelund, P. (1992). A bidimensional theory of achromatic color vision. Vision Research, 32, 2107-2119.

Hering, E. (1920). Grundzüge der Lehre vom Lichtsinn. Berlin: Springer-Verlag. [Translated by L. M. Hurvich \& D. Jameson (1964) as Outlines of a theory of the light sense. Cambridge, MA: Harvard University Press.]
KnAU, H. (2000). Thresholds for detecting slowly changing Ganzfeld luminances. Journal of the Optical Society of America A, 17, 13821387.

KozaKi, A. (1973). Perception of lightness and brightness of achromatic surface color and impression of illumination. Japanese Psychological Research, 15, 194-203.

KrAuSKOPF, J. (1963). Effect of retinal image stabilization on the appearance of heterochromatic targets. Journal of the Optical Society of America, 53, 741-744.

LADD-FrankLin, C. (1922). Tetrachromatic vision and the development theory of vision. Science, 55, 555-560.

LAND, E. H., \& McCANN, J. J. (1971). Lightness and retinex theory. Journal of the Optical Society of America, 61, 1-11.

Papaionnnou, J. (1974). Effects of retinal adaptation on brightness estimation in humans: Temporal considerations. Quarterly Journal of Experimental Psychology, 26, 514-519.

Quinn, P. C., Wooten, B. R., \& Ludman, E. J. (1985). Achromatic color categories. Perception \& Psychophysics, 37, 198-204.

Redding, G. M., \& Lester, C. F. (1980). Achromatic color matching as a function of apparent target orientation, target and background luminance, and lightness or brightness instructions. Perception \& Psychophysics, 27, 557-563.

Reid, R. C., \& Shapley, R. (1988). Brightness induction by local contrast and spatial dependence of assimilation. Vision Research, 28, $115-132$.

Ross, W. D., \& PessoA, L. (2000). Lightness from contrast: A selective integration model. Perception \& Psychophysics, 62, 1160-1181.

Rossi, A. F., \& PARAdiso, M. A. (1999). Neural correlates of perceived brightness in the retina, lateral geniculate nucleus, and striate cortex. Journal of Neuroscience, 19, 6145-6156.

Rossi, A. F., Rittenhouse, C. D., \& Paradiso, M. A. (1996). The representation of brightness in primary visual cortex. Science, $\mathbf{2 7 3}$, 1104-1107.

Shevell, S. K., Holliday, I., \& Whittle, P. (1992). Two separate neural mechanisms of brightness induction. Vision Research, 32, 2331-2340.

Sternheim, C. E., \& Boynton, R. M. (1966). Uniqueness of perceived hues investigated with a continuous judgmental technique. Journal of Experimental Psychology, 72, 770-776.

Sugita, Y. (1995). Contrast and assimilation on different depth planes. Vision Research, 35, 881-884.

Sugita, Y., \& Mimura, K. (1991). Cortical modulation of visual contrast. Psychological Research, 53, 271-273.

\section{NOTES}

1. White and black are the elemental components of achromatic colors (Heggelund, 1992; Hering, 1920; Ladd-Franklin, 1922; Quinn et al., 1985). The whiteness and blackness of an achromatic surface are, respectively, the perceived amount of white and of black in the color of the surface.

2. These results agree with those of Curtis and Rule (1978). Sternheim and Boynton's (1966) color-naming technique requires participants to express blackness and whiteness verbally in terms of percentages of black and white, respectively, without the constraint that the sum of these percentages has to be $100 \%$. Using this technique, Quinn et al. (1985) found that the percentage given for black is complementary to that given for white. This finding shows that the noncomplementarity of blackness and whiteness reported here could have depended on the response system.

(Manuscript received April 10, 2001; revision accepted for publication March 4, 2002.) 